\title{
Why doesn't the flow study work better for pulmonary atresia, ventricular septal defect, and major aortopulmonary collaterals?
}

\author{
James S. Tweddell, MD
}

\footnotetext{
From the Department of Cardiothoracic Surgery, The Heart Institute, Cincinnati Children's Hospital Medical Center; and the Department of Surgery, University of Cincinnati, Cincinnati, Ohio. Disclosures: Author has nothing to disclose with regard to commercial support.

Received for publication Aug 15, 2016; accepted for publication Aug 19, 2016; available ahead of print Sept 29, 2016.

Address for reprints: James S. Tweddell, MD, Heart Institute, Cincinnati Children's Hospital Medical Center, 3333 Burnet Ave, MLC 2013, Cincinnati, OH 45229-3026 (E-mail: James.Tweddell@ CCHMC.org).

J Thorac Cardiovasc Surg 2016;152:1504-5 $0022-5223 / \$ 36.00$

Copyright (C) 2016 Published by Elsevier Inc. on behalf of The American Association for Thoracic Surgery http://dx.doi.org/10.1016/j.jtcvs.2016.08.037
}

The management of patients with pulmonary atresia (PA), ventricular septal defect (VSD) and major aortopulmonary artery collaterals (MAPCAs) remains a challenge from both technical and decision-making standpoints. In this issue of The Journal of Thoracic and Cardiovascular Surgery, Zhu and colleagues ${ }^{1}$ from The Toronto Hospital for Sick Children have reviewed their recent experience with this challenging group of patients. They have adopted the use of a flow study, as initially described by Reddy and associates, ${ }^{2}$ to help with discriminate those patients in whom the VSD should be closed after unifocalization of the collaterals with the true pulmonary arteries. This analysis is a follow-up to their previous work with PA, VSD, and MAPCAs, in which they showed that the flow study had the highest accuracy at predicting successful VSD closure relative to other measures, such as the total neopulmonary artery index (TNPAI). ${ }^{3}$ The flow study is done intraoperatively with the patient on cardiopulmonary bypass after complete unifocalization and at the point in the operation just before the VSD closure. A second arterial cannula is placed in the pulmonary artery, and flow into the reconstructed pulmonary arteries is gradually increased until an index of 2.5 to $3.0 \mathrm{~L} / \mathrm{min} / \mathrm{m}^{2}$ is reached. When full flow is reached, the mean pulmonary arterial pressure is noted, and if this pressure is below a certain threshold it is believed to predict an acceptable postoperative right ventricular systolic pressure after VSD closure. Nevertheless, this is not a validation study of the previously derived criteria but rather a continued effort to identify the correct threshold. Indeed, in their original article that used the flow study, the threshold was $30 \mathrm{~mm} \mathrm{Hg}$, whereas in the current study Zhu and colleagues ${ }^{1}$ have meandered down to $25 \mathrm{~mm} \mathrm{Hg}$ and express concern when the pressure is between 25 and $30 \mathrm{~mm} \mathrm{Hg}$. Of more concern, values as low as $18 \mathrm{~mm} \mathrm{Hg}$ were associated with the need for VSD patch fenestration. Certainly this is a challenging group of patients, and any efforts to guide us

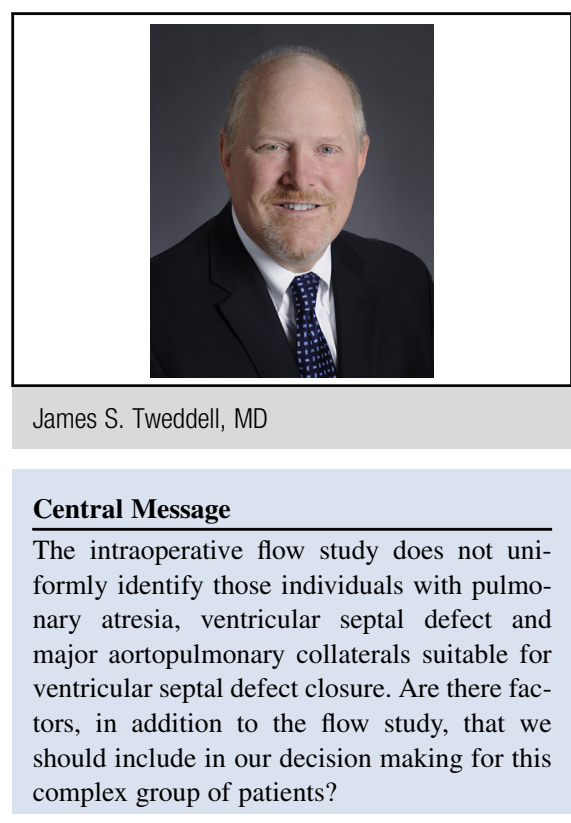

See Article page 1494.

are welcome; however, the flow study may be flawed because it assumes a relationship between the resistance in a nonpulsatile flow system, one that obeys Poiseuille's law, and the resistance obtained with pulsatile right ventricular derived flow. Even the best unifocalized pulmonary arterial tree of the patient with PA, VSD, and MAPCAs contains acute branch angles; abrupt caliber changes; elongated, tortuous vessels; and nondistensible anastomoses that are far from normal and will behave very differently with pulsatile circulation. In contrast to the intraoperative flow study, in vivo flow occurs during systole, which takes up only one-third of the cardiac cycle and will result in increased flow velocities relative to the steady-state flow study. Eddies and chaotic motion will result in turbulence, which will lead to increased resistance. Even with the reduced flow study pressure threshold of $25 \mathrm{~mm} \mathrm{Hg}$, the results were not entirely reassuring. RV failure was a contributing factor in 2 late deaths and was identified in $8 \%$ of survivors.

Zhu and colleagues ${ }^{1}$ chose to include nearly every patient in the study. Only 1 patient was excluded because of favorable anatomy. The inclusion of all of the patients, both those with higher TNPAI and those with less favorable anatomy, 
decreases the likelihood of finding the threshold value for the more challenging patient. As the data in this study suggest for patients with favorable anatomy and a TNPAI greater than $230 \mathrm{~mm}^{2} / \mathrm{m}^{2}$, the flow study does not add much value. Perhaps we need an algorithm that includes not only the flow study and TNPAI but also additional factors that might reasonably be thought to result in a difference between the flow study pulmonary arterial pressure and the real world RV pressure, including (1) the number of unifocalized collaterals and (2) the percentage of total pulmonary segments that these collaterals provide. These factors could be used to adjust for the complexity of the pulmonary arterial anatomy beyond in addition to the TNPAI and the flow study.

Ultimately the best outcome for patients with PA, VSD, and MAPCAs depends on, in addition to timely VSD closure, having as many lower pressure pulmonary segments as possible connected to the central pulmonary arteries without areas of stenosis or acute angulation. Zhu and colleagues ${ }^{1}$ are to be congratulated on their careful analysis of the flow study, identifying both the benefit and the limitations of this study for this complex and anatomically diverse population.

\section{References}

1. Zhu J, Meza J, Kato A, Saedi A, Chetan D, Parker R, et al. Pulmonary flow study predicts medium-term survival in pulmonary atresia with ventricular septal defect and major aortopulmonary collateral arteries. J Thorac Cardiovasc Surg. 2016; 152:1494-503.

2. Reddy VM, Petrossian E, McElhinney DB, Moore P, Teitel DF, Hanley FL. One-stage complete unifocalization in infants: when should the ventricular septal defect be closed? J Thorac Cardiovasc Surg. 1997;113:858-66; discussion $866-8$

3. Honjo O, Al-Radi OO, MacDonald C, Tran KC, Sapra P, Davey LD, et al. The functional intraoperative pulmonary blood flow study is a more sensitive predictor than preoperative anatomy for right ventricular pressure and physiologic tolerance of ventricular septal defect closure after complete unifocalization in patients with pulmonary atresia, ventricular septal defect, and major aortopulmonary collaterals. Circulation. 2009;120(11 Suppl):S46-52. 